\title{
Observations du vent de surface et des vagues à partir de CFOSAT
}

Le système d'observation spatiale de la Terre, composante essentielle des sciences océanographiques (Verron et Brasseur, 2017) vient de s'enrichir d'un nouvel élément : le satellite francochinois CFOSAT (China France Oceanography SATellite), lancé depuis la Chine avec succès le 29 octobre 2018 par une fusée Longue-Marche II, qui l'a placé en orbite polaire héliosynchrone à une altitude d'environ $520 \mathrm{~km}$.

CFOSAT a été conçu pour répondre aux besoins d'amélioration des connaissances concernant les caractéristiques de la surface océanique (vent, vagues) et leurs impacts sur les échanges entre l'atmosphère et l'océan qui jouent un rôle majeur dans le système climatique. De manière complémentaire aux autres observations spatiales actuelles, cette mission fournit des données essentielles pour la prévision atmosphérique, la prévision de l'état de la mer et la modélisation numérique du système océanatmosphère.

CFOSAT est original à plusieurs titres. D'une part, il s'agit de la première coopération dans le domaine de la recherche spatiale entre la France et la Chine. D'autre part, grâce à ses deux instruments radar, dont les concepts sont nouveaux, c'est une mission qui va permettre de mesurer en même temps et sur des zones communes le vent et les propriétés des vagues à la surface de l'océan. Elle complète ainsi de façon originale le système d'observation existant composé notamment de missions altimétriques (série Jason, Sentinel-3...), de missions embarquant des diffusiomètres vent (Ascat sur Metop par exemple) ou des radars imageurs (Sentinel-1 par exemple).

Jusqu'à mi-2019, les agences spatiales (Cnes, CNSA ${ }^{1}$, NSOAS ${ }^{2}$ ) et les laboratoires experts (en France le Latmos $^{3}$, le Lops ${ }^{4}$, l'équipe R\&D du Service de prévision marine de MétéoFrance) font porter tous leurs efforts sur la validation et l'étalonnage des instruments, puis sur la validation des variables géophysiques générées par les méthodes d'analyse mises au point dans les laboratoires de recherche. À l'issue de cette phase de validation, les données seront largement accessibles à la communauté scientifique.

\section{Instruments, principes de mesure et paramètres accessibles}

Le satellite embarque deux instruments radar fonctionnant autour de $2 \mathrm{~cm}$ de longueur d'onde (fréquence 13,4 GHz) : Scat (de Scatterometer), dédié à la mesure du vent de surface, conçu et développé en Chine, et Swim (Surface Waves Investigation and Monitoring), dédié à la mesure des propriétés détaillées des vagues, conçu et développé en France (figure 1).

\section{Swim}

Swim a été conçu pour la mesure du spectre directionnel des vagues, qui représente la répartition de la hauteur (ou de l'énergie) des vagues en fonction de leur direction de propagation et de leur longueur d'onde. À partir de ces spectres, de nombreux paramètres caractérisant les vagues peuvent être estimés, tels que la hauteur significative, la longueur d'onde et la direction de propagation dominantes, l'étalement de l'énergie des vagues en fonction de leur fréquence et de leur direction. Les spectres ainsi que les paramètres associés caractérisent l'évolution spatiale et temporelle des vagues générées par les systèmes météorologiques, qui se propagent loin de la zone source, en étant éventuellement affectées par les forçages locaux dus au vent, aux courants, à la bathymétrie, la glace de mer, etc.

Le principe de mesure utilisé pour Swim repose sur les propriétés de diffusion des ondes émises par un radar dans les conditions d'illumination de la surface marine proche de la visée nadir (voir Hauser et al., 2017). En effet, dans ces conditions, la diffusion des ondes électromagnétiques est principalement dominée par l'effet quasi spéculaire et donc très sensible à l'angle, dans le plan vertical, entre la direction de cette onde électromagnétique et la surface. Pour des angles d'incidence radar entre de 6 à $10^{\circ}$ par rapport au nadir, l'intensité rétrodiffusée est peu sensible aux variations de vent qui génère les vagues de courte longueur d'onde et servent de diffuseurs. En revanche, en présence de vagues de plusieurs dizaines à plusieurs centaines de mètres de longueur d'onde, l'intensité

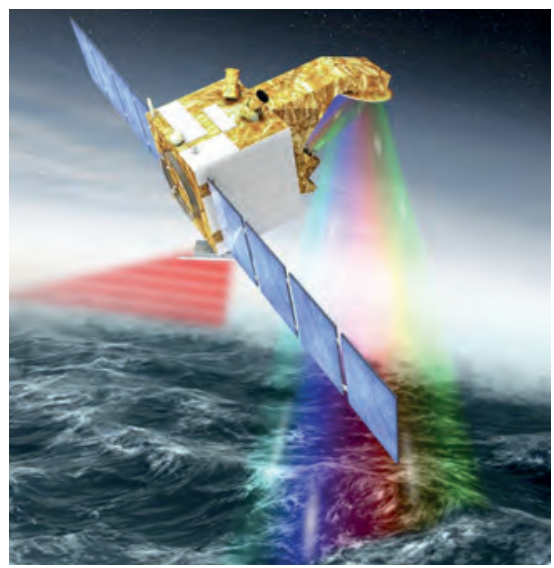

Figure 1. Vue schématique du satellite CFOSAT. Crédit : Cnes.

du signal renvoyé par la surface vers le radar est «modulée » par la pente locale de ces vagues longues. Ainsi, en utilisant un radar visant aux faibles incidences $(6$ à $10^{\circ}$ pour les mesures de vagues avec Swim), possédant une haute résolution spatiale dans la direction de visée $(0,5 \mathrm{~m}$ dans le cas de Swim) et illuminant une tache au sol suffisamment grande (environ $20 \mathrm{~km} \times 20 \mathrm{~km}$ dans le cas de Swim), on peut accéder à la répartition de la hauteur des vagues en fonction de leur longueur d'onde dans la direction d'observation. En utilisant de plus un faisceau radar tournant sur $360^{\circ}$ d'azimut autour de l'axe vertical, on accède au spectre directionnel des vagues. Le principe de mesure repose donc sur l'analyse des variations d'intensité rétrodiffusée par la surface à l'intérieur de chaque « tache au sol » illuminée par le radar. Compte tenu de la géométrie choisie, l'échelle spatiale à laquelle les spectres directionnels de vagues peuvent être estimés à partir de Swim est de l'ordre de $70 \mathrm{~km} \times 90 \mathrm{~km}$ de part et d'autre de la trace satellite. Les vagues qui peuvent être analysées sont celles de longueur d'onde comprises entre 70 à $500 \mathrm{~m}$, et la précision de mesure attendue est d'environ $10 \%$ sur la longueur d'onde, $15^{\circ}$ sur la direction de propagation et $20 \%$ sur l'énergie. La phase de validation géophysique qui a débuté devra confirmer si les performances observées sont conformes à celles attendues.

\footnotetext{
1. China National Space Administration

2. National Satellite Ocean Application Service

3. Laboratoire atmosphère, milieux, observations spatiales

4. Laboratoire d'océanographie physique et spatiale
} 
Ce concept de mesure, utilisé pour la première fois dans l'espace, permet à Swim d'être très complémentaire des autres observations de l'état de la mer depuis l'espace. En effet, les mesures fournies par les missions altimétriques ne fournissent que la hauteur significative des vagues, donc aucune information sur leur répartition spectrale. De leur côté, les radars imageurs (Synthetic Aperture Radar ou SAR) peuvent fournir des informations spectrales, mais seulement pour les plus grandes longueurs d'onde (supérieures à 200 ou $250 \mathrm{~m}$ ) en raison du principe d'imagerie radar qui utilise non seulement l'intensité mais également la phase du signal, cette dernière étant fortement affectée au-dessus de l'océan par le mouvement des vagues qui crée un effet de filtrage limitant ou distordant la détection des vagues.

\section{Scat}

Linstrument Scat repose sur un concept utilisé de façon régulière depuis les années 1990 : à incidence moyenne (typiquement de 20 à $50^{\circ}$ par rapport au nadir), la diffusion des ondes électromagnétiques est très sensible à la rugosité de surface créée par les vagues courtes (de quelques centimètres) générées par le vent local. Plus le vent augmente, plus l'intensité reçue par le radar est importante. Cette rétrodiffusion est fortement anisotrope (maximum de rétrodiffusion dans la direction face et dos au vent, minimum dans les directions perpendiculaires). Ainsi, en utilisant un système visant selon plusieurs directions azimutales, l'intensité et la direction du vent peuvent être estimées. L'originalité du Scat de CFOSAT est qu'il combine les avantages des deux concepts jusqu'ici mis en œuvre depuis l'espace : celui avec une géométrie à large fauchée mais antennes fixes (tel qu'utilisé pour l'instrument Ascat de Metop) et celui avec une géométrie à faisceau radar étroit mais balayant sur $360^{\circ}$ (tel qu'utilisé sur la mission Quickscat américaine ou Oscat indienne). Le concept du Scat de CFOSAT offre un bon compromis entre échantillonnage spatial pour l'estimation du vent et levée d'ambiguïtés sur la direction du vent. Les données de vent issues du Scat seront fournies par la Chine avec un maillage de $25 \mathrm{~km} \times 25 \mathrm{~km}$ sur une fauchée d'environ $500 \mathrm{~km}$ de part et d'autre de la trace satellite.

\section{Premières analyses, premiers travaux scientifiques attendus}

Pendant la phase actuelle de vérification (dite phase CAL/VAL) qui va s'étaler jusqu'à sept mois après le lancement, trois laboratoires experts en France et 1'entreprise CLS travaillent en étroite relation avec le Cnes pour contrôler et affiner les méthodes d'inversion, quantifier les incertitudes sur les variables géophysiques estimées, éventuellement corriger des biais d'estimation. Un sous-ensemble de données du radar Swim est accessible à ces équipes depuis fin novembre 2018 (exemples en figures 2 et 3 ). Les informations utilisées comme référence pour cette phase de validation statistique des données Swim proviennent de mesures in situ (bouées), des modèles de prévision d'état de mer (modèles de prévision des vagues MF-WAM de Météo-France, modèle WW3, modèle WAM du Centre européen pour les prévisions météorologiques à moyen terme), des données d'autres satellites (altimètres, SAR...).

Au-delà des travaux de validation, la communauté internationale se mobilise pour proposer des projets de recherche, comme en atteste la trentaine de réponses reçues à l'appel à idées diffusé mi-2018. Les sujets proposés sont très variés : hydrodynamique des vagues, rôle des vagues dans les échanges océan/atmosphère et la dynamique océanique, compréhension et caractérisation d'événements extrêmes (tempêtes, cyclones tropicaux), et de leurs impacts, interactions entre vagues et glace de mer en zone polaire, impact des observations au large pour mieux caractériser les conditions côtières, assimilation d'observations dans les modèles numériques, sans oublier les études sur la glace de mer et les surfaces continentales (végétation, a)

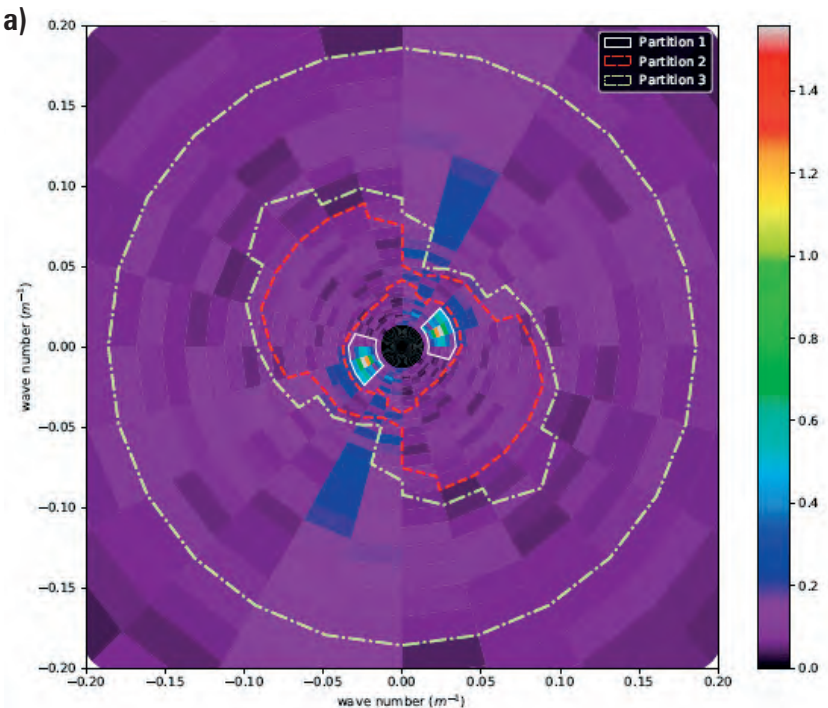

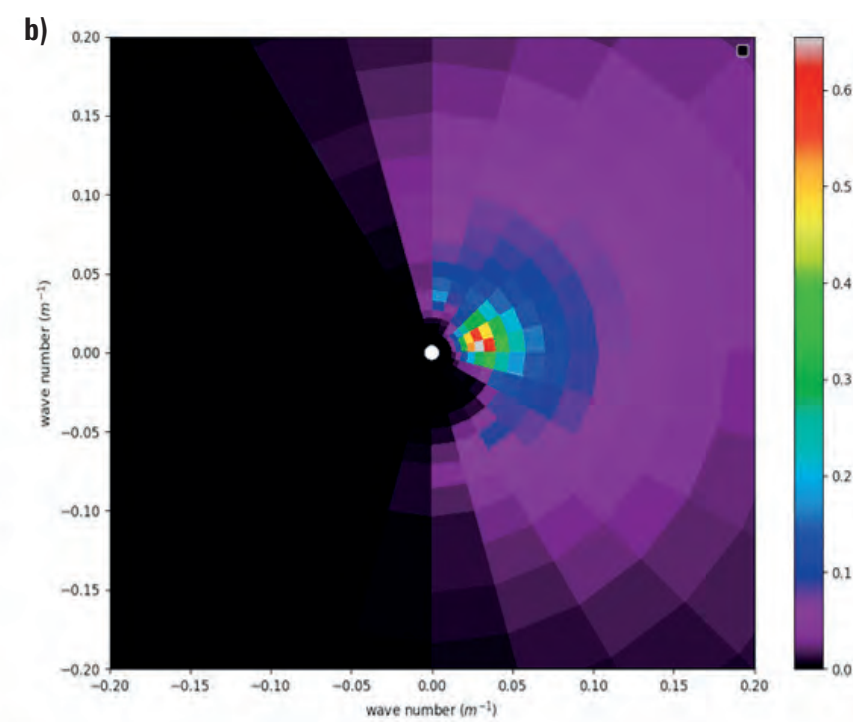

Figure 2. (a) Spectre directionnel de pente des vagues estimé à partir des observations du radar Swim à $8^{\circ}$ d'incidence, comparé au (b) spectre directionnel issu du modèle de vagues MF-WAM. Sur ces figures en représentation polaire, la couleur représente la densité spectrale, l'angle par rapport au haut de la page représente la direction de propagation, et la distance par rapport au centre est inversement proportionnelle à la longueur d'onde $\lambda$ (l'échelle des nombres d'ondes $k=2 \pi / \lambda$ est indiquée sur les axes $x$ et $y$ ). Cet exemple est associé à une mesure de Swim au-dessus du Pacifique Sud le 20 novembre $2018\left(49,6^{\circ}\right.$ S, 102,5 W) vers 13 h 00 TU. Sur la figure de gauche, la partition la plus énergétique est entourée par des traits continus blancs. Elle est en bon accord avec le spectre de MF-WAM. Le reste du spectre de Swim apparaît comme essentiellement du bruit. Ce bruit était attendu avant le lancement (bruit de speckle), mais difficilement quantifiable par des simulations. Les travaux sont en cours pour mieux éliminer cet effet. 

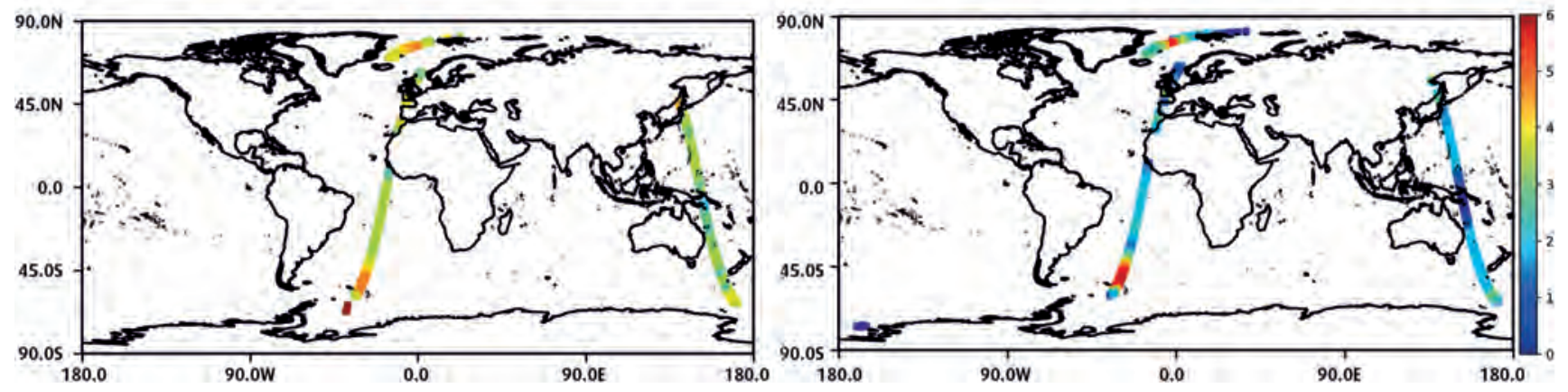

Figure 3. Hauteur significative des vagues déduite de la mesure du radar Swim à $8^{\circ}$ d'incidence (à gauche) comparée à celle fournie par le modèle de vagues du Centre européen pour les prévisions météorologiques à moyen terme (à droite). Bien que les valeurs ne soient pas exactement identiques, ces figures montrent que les données Swim permettent de restituer une dynamique globalement cohérente avec celle du modèle. Des travaux sont en cours pour mieux prendre en compte les erreurs de mesure (bruit de speckle notamment) et permettront de rapprocher les deux informations.

humidité, rugosité) qui pourront être abordées à partir de l'analyse multidirectionnelle et multi-incidence des coefficients de rétrodiffusion radar. Une fois validées, les données de vagues et de vent viendront également alimenter les systèmes opérationnels, en premier lieu ceux de Météo-France, ainsi que le service européen Copernicus. Les récents travaux de Météo-France sur l'assimilation de données simulées et celles provenant du SAR de Sentinel-1 indiquent tout l'intérêt de cette assimilation d'informations spectrales de vagues. Le système d'assimilation qui ingère depuis décembre 2018 des données SAR de Sentinel-1 va donc s'enrichir prochainement des données CFOSAT.

Danièle Hauser

Laboratoire atmosphères, milieux, observations spatiales Institut Pierre-Simon-Laplace

Hauser D., Tison C., Amiot T., Delaye L., Corcoral N., Castillan P., 2017. SWIM: the first spaceborne wave scatterometer. IEEE Trans. Geosci. Rem. Sens., 55, 3000-3014. doi: 10.1109/TGRS.2017.2658672

Verron J., Brasseur P., 2017. Apports de l'observation satellitaire à l'océanographie. La Métérorologie, 97, 62-69.

\section{lasi sur Metop-C : du lancement à la première mesure}

Iasi (Interféromètre atmosphérique de sondage infrarouge) est un interféromètre de Michelson couvrant le domaine spectral infrarouge $\left(645-2760 \mathrm{~cm}^{-1}\right.$ en nombre d'onde ou 3,62 à 15,5 $\mu \mathrm{m}$ en longueur d'onde). Conçu par le Cnes, construit par Thalès Alenia Space et opéré par Eumetsat, cet instrument est destiné au sondage atmosphérique à haute résolution spectrale. Il fournit des informations sur la structure verticale de la température et de l'humidité atmosphériques, avec une précision sans précédent et une résolution verticale de $1 \mathrm{~km}$. Il surveille aussi les concentrations atmosphériques de gaz à l'état de traces et de gaz à effet de serre, comme l'ozone, le monoxyde de carbone et le dioxyde de soufre.

L'instrument est embarqué sur trois plateformes satellitaires Metop (Meteorological Operation) qui forment le segment spatial du programme polaire d'Eumetsat (EPS pour Eumetsat Polar System). Les satellites Metop ont été développés dans le cadre d'un programme de l'Agence spatiale européenne. Metop- $A$ et Metop-B ont été lancés de Baïkonour respectivement les 19 octobre 2006 et 17 septembre 2012 ; ils fournissent des données opérationnelles depuis juillet 2007 et avril 2013. Le troisième satellite qui complète le système polaire d'Eumetsat a été lancé avec succès depuis la Guyane française le 7 novembre 2018 à 1 h 47 TU ( 21 h 47 heure de Kourou) par le Soyouz ST-B. Chaque satellite embarque une version identique de l'instrument Iasi.

Entre le lancement du satellite, les premières mesures de Iasi et la mise en œuvre opérationnelle de l'instrument pour dissémination, de nombreuses étapes sont nécessaires.

Quelque 60 minutes après le lancement, l'étage supérieur du Soyouz a placé Metop- $C$ sur orbite. Le contact avec ce dernier a été établi par l'European Space Operation Center (Esoc) grâce à la station au sol de Yatharagga en Australie. Pendant les premiers jours, les équipes de l'Esoc ont eu pour mission de positionner le satellite à proximité de son orbite finale avant de céder progressivement et complètement le contrôle (hand-over) à Eumetsat. Les équipes « instruments », telles que le Cnes pour Iasi sur Metop$C$ (par la suite, Iasi-C) démarrent ensuite la recette fonctionnelle du sondeur depuis le centre de contrôle des satellites Metop à Darmstadt.

Trente-trois jours après le lancement et après avoir passé avec succès un ensemble d'opérations amenant Iasi-C aux conditions nominales d'opérations (allumage, décontamination des détecteurs, libération des coins de cube, etc.), l'instrument livre les premiers interférogrammes le 10 décembre 2018 (données d'entrée pour le calcul des spectres) qui attestent du bon fonctionnement de l'interféromètre (figure 1).

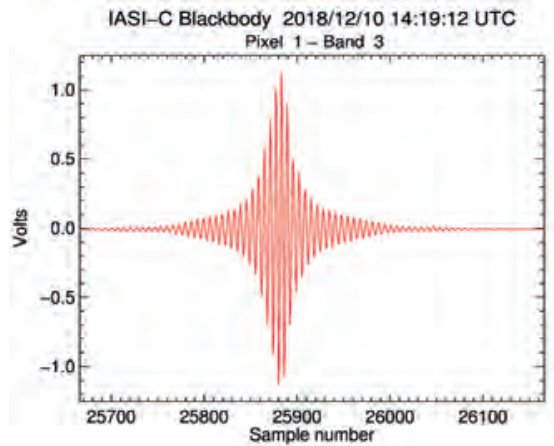

Figure 1. Premier interférogramme de lasi sur Metop-C produit par l'équipe scientifique Hyperspectral à Eumetsat, avec la collaboration du Cnes. Source : http://scienceblog.eumetsat.int 